\title{
Why pebbles float to the surface
}

The reasons why mixtures of sand and pebbles seem to separate when shaken vigorously have been illuminated by an intriguing simulation which, among other things, suggests that only large pebbles behave in this way.

$\mathrm{WHY}_{\mathrm{HY}}$ is it that when you shake a pail containing, say, a mixture of sand and pebbles, the larger pebbles tend to rise to the top? In this, the Northern Hemisphere's summer, the observation must be repeated countless times each day by untrained observers, mostly infants, some just able to walk, playing on crowded beaches. It should therefore be of some comfort to them (or to those among their parents who believe that children's questions should be answered whenever possible) that an explanation of the phenomenon has now been given by three French researchers - Rémi Jullien and Paul Meakin from the University of Paris-Sud and André Pavlovich from the Saclay Centre at Gif-sur-Yvette in the Paris suburbs (Phys. Rev. Lett. 69, 640; 27 July 1992).

Naturally a phenomenon of such universal interest needs a name: 'segregation by shaking'. But Jullien and his colleagues note that shaking is merely one of the several industrially important ways in which particles of different sizes are separated from each other mechanically - simple pouring will often decant the smaller particles preferentially, vibration will do the trick, and so on.

But how to model a complicated phenomenon such as the shaking of a mixture of particles of different sizes? On the face of things it is a formidable task requiring that all the particles in an assemblage should be handled separately. That is what Jullien and his colleagues have done or, rather, have arranged that their computers should do for them. The recipe is almost as simpleminded as it could be.

The bottom of the pail is represented by a flat horizontal surface. To begin with, spherical particles of different sizes are figuratively dropped on to it in random order by means of vertical trajectories also chosen randomly. The rules are that particles hitting the flat surface keep their positions, losing their momentum instantaneously. Eventually the time comes when the figuratively falling particles do not hit the flat surface itself, but fall on top of other particles, when the rules of the simulation require that they follow the steepest possible downward path until they reach the lowest point accessible from their place of impact. When that happens, the fallen particles are irreversibly incorporated into the growing pile of sand and pebbles. That accounts for the formation of what might be called the

\section{pail's first charge.}

The next step is to simulate the shaking, which is done in the simplest way possible. The trick is to list all the figurative particles in the simulation in order of their increasing height above the flat surface (where the height is that of the centre of the sphere), together with a note of their coordinates on that surface. Next, the whole assemblage is made figuratively to fall again, each particle along a trajectory defined by its original coordinates and, crucially, with the lowest particles falling first. Otherwise, the rules of the simulation remain the same; particles large or small hitting the bottom of the simulated pail or the growing pile that covers it lose their momentum once they reach the lowest position accessible from their impact point. The process is then repeated time and time again until the larger particles (the pebbles) are all lying on or near the surface of the pile.

It is interesting that the process as modelled is simply geometrical. Neither the physical energy imparted to the particles by shaking nor the relative density of the large and small particles enters into the simulation. Moreover, the rules of the simulation do not allow the weight of the larger spheres to displace smaller particles on which they rest from their positions, so that the simulation does not even provide for local static equilibrium.

Even so, what Jullien and his colleagues have done provides a simply explanation of why the larger particles seem to float to the surface on shaking. Smaller particles lying near the bottom of the pail at one iteration will inevitably lie near the bottom at the next, perhaps more compactly so. The larger spheres, on the other hand, will inevitably be dropped relatively later (because their turn to drop will be delayed until the simulation has reached the height represented by the position of their centres) and will then have to find a resting place on a substratum of smaller spheres already in place. At successive iterations they can move up but not down.

Jullien and his colleagues have carried through endless simulations. For example, they illustrate their article with the results of numerical experiments with 50,000 small spheres and 250 larger spheres whose diameters are four times as great. Sixty shakes (as defined) are enough to bring most of the larger spheres to the surface. Crosssections through the simulated assemblage at different stages show the larger spheres resting on smaller spheres (three-point contact is enough, under the rules, to support each of them) in such a way that there are also substantial voids beneath them. Perhaps the simple way of explaining segregation by shaking is that the ratchet that drives the larger spheres only upwards arises from the tendency of smaller spheres to occupy these voids on successive shakings.

At least roughly, it is even possible to calculate what the upward velocity of one of the larger spheres should be. If the size disparity between large and small spheres is great enough (and ideally infinite), the void beneath a large particle will be conical in shape with an angle represented by the angle of repose of the smaller spheres - the angle of the slope of a conical heap of them, for example.

Iterations of the simulation then coat the conical void with a uniform layer of the smaller spheres consisting of all those that would have been dropped before the larger spheres simply because their centres were lower down. The prediction of this calculation agrees with the results of the simulation.

The much more intriguing conclusion is that there is a kind of critical phenomenon involved in segregation by shaking. The process seems to function if the ratio of the diameters of the large and small particles is greater than a certain value, estimated at 2.8 .

From markedly greater disparities of size, the efficiency of the segregation seems independent of the ratio of the two diameters, although the upward velocity of very large particles is less than that of particles that are slightly smaller. But when the radii of the large particles are less than 2.8 times the radii of the smaller particles with which they are mixed, it seems that the larger particles move upwards only by a finite distance and then, statistically, come to rest.

Jullien and his colleagues are properly eager to point out the limitations of their simulation - its neglect of friction, energy and all that. But they are surely correct to argue that a recognition of this critical phenomenon must be of some practical significance in industry. It may also divert many of those who now find themselves marooned on beaches in the Northern Hemisphere.

John Maddox 